\author{
Pasquale Nascenti \\ (insegnante di religione cattolica nella scuola pubblica)
}

\title{
Un'intesa particolare: elementi procedimentali non concordatari in vista del concorso per insegnanti di religione*
}

\author{
A particular agreement: procedural elements not agreed upon next contest \\ for teachers of the Catholic religion
}

\begin{abstract}
This paper aims to examine the agreement signed on December 14, 2020 between the Minister of Education and the President of the Italian Bishops' as an act prior to a contest for teachers of the Catholic religion. In light of the previous agreements and some specific studies, the author attempts to highlight how some elements of State competence have merged into a procedural act, wondering if this represents an overrun of the respective prerogatives.

SOMMARIO: 1. Premessa generale: il quadro normativo di riferimento - 2 . Un'intesa particolare - 3. La certificazione dell'idoneità diocesana - 4. La riserva dei posti messi a bando - 5. Il programma d'esame - 6. La composizione delle commissioni giudicatrici - 7. Conclusioni.
\end{abstract}

\section{1 - Premessa generale: il quadro normativo di riferimento}

L'insegnamento della religione cattolica (IRC) nelle scuole italiane è tema di ampia portata che per la peculiarità della sua natura di res mista investe pienamente le parti interessate, quella statale e quella ecclesiale, e, di conseguenza, si pone come materia di studio (anche) per il diritto ecclesiastico. ${ }^{1}$

* Contributo sottoposto a valutazione.

1 Per un'introduzione generale all'IRC si veda: S. CICATELLI, Prontuario giuridico IRC. Raccolta commentata delle norme che regolano l'insegnamento della religione cattolica nelle scuole di ogni ordine e grado, $7^{\text {a }}$ ed., Queriniana, Brescia, 2012; AA. VV. (a cura di S. CiCATELli, G. MALIZIA), Una disciplina alla prova. Quarta indagine nazionale sull'insegnamento della religione cattolica in Italia a trent'anni dalla revisione del Concordato, Elle Di Ci, Torino, 2016. Per un approccio più strettamente storico si veda: E. BUTTURINI, La religione a scuola. Dall'Unità ad oggi, Queriniana, Brescia, 1987; AA. VV. (a cura di L. CAIMI, G. VIAN), La 
L'assetto istituito con l'art. 36 del Concordato del 1929 era stato attuato dalla legge n. 824 del 1930, norma che ha di fatto retto l'insegnamento per più di un cinquantennio e le cui tracce sono, nonostante l'abrogazione dell'intero provvedimento nel 2008, ancora oggi rinvenibili negli artt. 309-310 del Testo unico della scuola d.lgs. n. 297 del 1994. L'art. 9.2 dell'Accordo di Revisione del 1984, ratificato con legge n. 121 del 1985, ha profondamente mutato la natura dell'insegnamento che da "fondamento e coronamento dell'istruzione pubblica" diviene disciplina "inserita nel quadro delle finalità della scuola". In relazione al medesimo articolo, il Protocollo addizionale stabiliva gli elementi sui quali le due autorità, il Ministero dell'Istruzione e la Conferenza episcopale italiana, devono addivenire a un comune accordo tramite specifica intesa. Tali elementi sono: 1) i programmi di insegnamento per i diversi ordini e gradi delle scuole pubbliche, 2) le modalità di organizzazione dell'insegnamento, anche in relazione alla collocazione nel quadro degli orari delle lezioni, 3) i criteri per la scelta dei libri di testo, 4) i profili di qualificazione professionale degli insegnanti. Tre sono state le intese finora sottoscritte dalle autorità competenti: d.p.r. n. 751 del 1985, d.p.r. n. 202 del 1990, d.p.r. n. 175 del $2012^{2}$.

La legge n. 186 del 2003, norma volta a stabilizzare i docenti attraverso l'introduzione di un apposito status giuridico, ha apportato degli elementi di novità rispetto all'assetto bilaterale, pur senza stravolgerlo3. L'istituzione di "due distinti ruoli regionali,

religione istruita. Nella scuola e nella cultura dell'Italia contemporanea, Morcelliana, Brescia, 2013. Per i rilievi più pertinenti agli studi ecclesiasticistici si veda: $\mathbf{M}$. MADONNA, Lo status giuridico degli insegnanti di religione cattolica tra diritto della Chiesa e ordinamento dello Stato, Libellula, Lecce, 2018; A. BETTETINI, Lo status giuridico degli insegnanti di religione cattolica, in Stato, Chiese e pluralismo confessionale, Rivista telematica (https://www.statoechiese.it) n. 39 del 2012.

2 Sull'ultima Intesa, cui ha dato esecuzione il D.P.R. n. 175 del 2012, che modifica notevolmente le precedenti, si veda: S. ATTOLINO, La nuova qualificazione dei docenti di religione: note al margine del D.P.R. n. 175 del 2012, in Stato, Chiese e pluralismo confessionale, cit., n. 41 del 2013.

3 Sull'introduzione del ruolo per i docenti di religione e sul periodo di assestamento che ne è derivato si veda: S. CICATELLI, Lo stato giuridico è legge, in Religione\&Scuola, 2003, 1, p. 5 ss.; P. CONSORTI, Sul nuovo stato giuridico degli insegnanti di religione cattolica, con particolare riferimento alla loro mobilità, in Stato, Chiese e pluralismo confessionale, cit., giugno 2009; M. GATTAPONI, Insegnanti di religione: dalla precarietà all'immissione in ruolo. Primi nodi al vaglio della Corte 
articolati per ambiti territoriali corrispondenti alle diocesi" (art. 1, primo comma) ha necessariamente comportato la definizione delle modalità di accesso ai medesimi, nella fattispecie "previo superamento di concorsi per titoli ed esami, intendendo per titoli quelli previsti dall'Intesa" (art. 3, primo comma), fermi restando i requisiti stabiliti dalla normativa concordataria per l'insegnamento e cioè, oltre ai titoli di studio, anche il possesso "del riconoscimento di idoneità [...] rilasciato dall'ordinario diocesano competente per territorio" (art. 3, quarto comma). L'indizione dei concorsi fu fissata, almeno nelle intenzioni della norma, con frequenza triennale (art. 3, secondo comma). Tra le disposizioni transitorie e finali la legge previde che il primo concorso per titoli ed esami fosse riservato agli insegnanti di religione che avessero prestato continuativamente servizio per almeno quattro anni nel corso degli ultimi dieci anni (art. 5, primo comma).

La frequenza regolare di un concorso per l'immissione in ruolo degli insegnanti di religione è stata completamente disattesa negli ultimi anni e l'approfondimento delle ragioni che hanno portato al conseguente ingigantimento del precariato esula da questo contributo. ${ }^{4}$

\section{2 - Un'intesa particolare}

Il d.1. n. 126 del 2019 convertito con modificazioni dalla legge n. 159 del 2019, ha introdotto, con l'art. 1-bis, disposizioni urgenti in materia di reclutamento del personale docente di religione cattolica. Con il primo comma il legislatore autorizza il Ministro dell'Istruzione

"a bandire, previa intesa con il Presidente della Conferenza episcopale italiana, un concorso per la copertura dei posti per l'insegnamento della religione cattolica che si prevede siano vacanti e disponibili negli anni scolastici dal 2020 del 2021 al 2022 del 2023" 5 .

Costituzionale, in Quaderni di diritto e politica ecclesiastica, 2006, 3, p. 785 ss.

${ }^{4} \mathrm{Cfr}$. P. NASCENTI, Uno stallo indefinito. Il reclutamento dei docenti di religione cattolica: status quo e prospettive, in Dirigenti Scuola, 2020, 3, p. 200 ss.; ID., Quale concorso? I precari e la recente Intesa tra Stato e CEI, in Il Regno, 2021, 4, p. 79 ss.

5 Il d.1. 'Milleproroghe' n. 183 del 2020, convertito con modificazioni dalla legge n. 21 del 2021, con l'art. 6, primo comma, ha prorogato l'autorizzazione a 
Il complesso stato di emergenza causato dalla pandemia da Covid-19 ha contribuito al rallentamento, ma non al blocco, dei passi successivi. Nel giugno del $2020^{6}$ si è insediato un Tavolo di lavoro congiunto tra il Ministero dell'Istruzione e la Conferenza episcopale italiana che si è posto tra gli obiettivi anche la definizione dell'intesa prevista dall'art. 1-bis della legge n. 159 del 2019. Il 14 dicembre 2020 l'intesa è stata formalizzata con la firma delle autorità competenti, il Card. Gualtiero Bassetti, Presidente della Conferenza episcopale italiana, e l'On. Lucia Azzolina, Ministra dell'Istruzione ${ }^{7}$. Il presente contributo si propone di approfondire questa intesa, provando a definirne la natura alla luce della normativa generale e concordataria ed esplorandone la (eventuale) portata di res mixta, individuando alcune difformità rispetto al quadro normativo.

L'intesa è composta da una premessa e sette articoli di modesta ampiezza; in allegato alla stessa troviamo il d.m. n. 70 del 2020 e l'annesso elenco delle discipline ecclesiastiche, delle facoltà e degli istituti abilitati a rilasciare titoli di studio che costituiscono qualificazione professionale valida per l'IRC. Il seguente schema ne sintetizza i contenuti:

\begin{tabular}{|l|l|}
\hline Premessa & $\begin{array}{l}\text { Richiamo alle precedenti intese: d.p.r. n. 751 del 1985, } \\
\text { d.p.r. n. 202 del 1990, d.p.r. n. 175 del 2012 e all'art. 1-bis } \\
\text { della legge n. 159 del 2019 }\end{array}$ \\
\hline Art. 1 & $\begin{array}{l}\text { Quadro normativo di riferimento per parte concordataria: } \\
\text { legge n. 121 del 1985, d.p.r. n. 175 del 2012 }\end{array}$ \\
\hline Art. 2 & $\begin{array}{l}\text { Quadro normativo di riferimento per parte statale: legge } \\
\text { n. 186 del 2003, legge n. 159 del 2019, normativa statale } \\
\text { sui concorsi pubblici }\end{array}$ \\
\hline Art. 3 & $\begin{array}{l}\text { Titoli di qualificazione professionale per partecipare al } \\
\text { concorso }\end{array}$ \\
\hline Art. 4 & Certificazione dell'idoneità diocesana \\
\hline Art. 5 & $\begin{array}{l}\text { Riserva dei posti messi a bando } \\
\text { Art. 6 }\end{array}$ \\
$\begin{array}{l}\text { Definizione di alcuni elementi facenti parte del bando di } \\
\text { concorso }\end{array}$ \\
\hline Art. 7 & $\begin{array}{l}\text { Elementi del programma d'esame e definizione della } \\
\text { composizione delle commissioni d'esame del concorso }\end{array}$ \\
\hline
\end{tabular}

bandire per il triennio 2021-2024.

${ }^{6}$ Insegnamento religione cattolica: al via Tavolo Miur-Cei, in SIR (www.agensir.it), 19 giugno 2020.

7 Scuola, Intesa Ministero-CEI su concorso per Insegnanti Cattolica, (www.miur.gov.it), 14 dicembre 2020. 
Data la materia di cui tratta, cioè una procedura concorsuale per dipendenti statali, non è plausibile assimilare questa intesa alle precedenti (1985, 1990, 2012), le quali erano più facilmente e direttamente inquadrabili come sub-concordatarie, prodotto diretto di un accordo internazionale, sia per le materie in esse trattate, le quali rientravano tra quelle elencate nell'Accordo di revisione, sia per la forma del d.p.r. che le caratterizzò e che aveva così permesso di produrre effetti nell'ordinamento italiano. $\mathrm{Va}$, però, puntualizzato che la richiesta di una tale intesa è una novità decisamente assoluta: non si riscontra nella normativa concordataria e nella normativa statale riguardante $1^{\prime} I R C$ e lo stato giuridico dei docenti, la necessità di un'intesa del genere. Il bando dell'unica procedura concorsuale per insegnanti di religione cattolica $^{8}$ finora svoltasi in Italia non era stato preceduto da nessuna intesa, né la legge n. 186 del 2003 vi faceva riferimento come atto che in qualche modo dovesse precedere un bando.

Le due autorità coinvolte si erano, però, già in precedenza trovate a stipulare specifici accordi in altri campi. Ci si riferisce, ad esempio, a quelli riguardanti il settore dei beni culturali ma, a differenza dell'intesa del dicembre 2020, essi non sono stati sottoscritti tra il Presidente della Conferenza episcopale italiana e il Ministro dell'Istruzione ma da responsabili degli organi del Ministero, e, su autorizzazione del Presidente, dai direttori degli uffici della Conferenza episcopale competenti per materia. Si tratta, però, di forme di collaborazione previste dall'art. 12 dell' Accordo di revisione "per la tutela del patrimonio storico e artistico" 9.

Esempi similari sono anche quegli accordi nati a livello locale, stipulati tra le Conferenze episcopali regionali e le Regioni, favoriti dalla riforma del Titolo $\mathrm{V}$ della Costituzione introdotta dalla legge costituzionale n. 3 del 2001. In una prospettiva partecipativa e di sussidiarietà, tema centrale nella sentenza n. 303 del 2003 della Corte costituzionale, il riformato art. 118 assegna alle Regioni, alle Province e ai Comuni l'autonoma iniziativa amministrativa per lo

8 Decreto del Direttore Generale del personale della scuola del Dipartimento per l'istruzione del Ministero dell'istruzione, dell'università e della ricerca del 2 febbraio 2004 pubblicato nella Gazzetta Ufficiale, $4^{\circ}$ serie speciale, n. 10 del 6 febbraio 2004

${ }^{9}$ Cfr. I. BOLGIANI, I beni culturali di interesse religioso tra Intesa nazionale $e$ accordi regionali ("vecchi" e "nuovi"), in Stato, Chiese e pluralismo confessionale, Rivista telematica (www.statoechiese.it), n. 33 del 2012. 
svolgimento di attività di interesse generale, aprendo a forme di cooperazione con soggetti terzi ${ }^{10}$.

L'intesa sottoscritta a dicembre non sembra allinearsi agli esempi sopra accennati, non soltanto per la diversità della materia trattata ma soprattutto per la diversità delle parti stipulanti: non autorità locali o articolazioni periferiche della Conferenza episcopale e del Ministero ma Presidente e Ministro in persona.

La norma predisposta dal legislatore con 1'art. 1-bis, che ha collocato l'intesa come previa al bando, il quale è atto essenzialmente amministrativo, la rende, per alcuni aspetti, simile agli accordi procedimentali ex art. 11 della legge n. 241 del 199011, dove viene utilizzato lo strumento dell'accordo concertato allo scopo di contemperare gli interessi dei soggetti coinvolti nei procedimenti, assicurando preventivamente il consenso delle parti, per un migliore perseguimento dell'interesse pubblico. Tali accordi, stipulati per iscritto a pena di nullità, sono condotti senza pregiudizio verso terzi e assoggettati, nel caso di contenzioso, alla giustizia amministrativa. Si tratta, comunque, di un accostamento e non di piena sovrapponibilità tra l'intesa di dicembre e l'accordo come descritto ex art. 11, poiché l'art. 13 della medesima norma ne esclude l'applicabilità nei casi dell'attività della Pubblica amministrazione diretti all'emanazione di atti amministrativi generali.

Non desta meraviglia, quindi, che ci si trovi di fronte a un atto ibrido e che tale ibridazione vada forse collocata sullo sfondo del più generale ambito di cooperazione democratica tra Stato e formazioni sociali che consente di ricorrere a poliedriche e flessibili forme convenzionali, opportune e calibrate, nella fase di sviluppo di procedimenti e norme di competenza statale ${ }^{12}$.

${ }^{10}$ Cfr. A. BETTETINI, Tra autonomia e sussidiarietà: contenuti e precedenti delle convenzioni a carattere locale tra Chiesa e Istituzioni pubbliche, in Stato, Chiese e pluralismo confessionale, cit., maggio 2010. Cfr. anche G. ROSSANO, Le fonti del diritto e il principio di sussidiarietà nel quadro dei più recenti interventi legislativi per la "semplificazione", in Diritto amministrativo, 2001, 9, pp. 273-308; D. MILANI, La tutela degli interessi religiosi delle comunità locali tra riforma della Costituzione e nuovi statuti regionali, in Quaderni di diritto e politica ecclesiastica, 2005, 1, pp. 201244.

11 Cfr. M. FRATINI, Manuale sistematico di diritto amministrativo, Dike Giuridica Edizioni, Roma, 2008, pp. 872-875.

12 Cfr. N. BASSI, Accordi amministrativi verticali e orizzontali: la progressiva ibridazione dei modelli, in M. CAMMELLI, Territorialità e delocalizzazione del 
Le esplorazioni su esposte conducono a chiederci se questa intesa procedimentale di concerto amministrativo possa collocarsi all'ombra dell'art. 7 della Costituzione, se possa, cioè, essere investita dagli obblighi bilaterali derivanti dal Concordato.

La possibilità di concordare su ulteriori materie era stata già prevista con l'art. 13.2 dell'Accordo di Revisione:

“ulteriori materie per le quali si manifesti l'esigenza di collaborazione tra la Chiesa cattolica e lo Stato potranno essere regolate sia con nuovi accordi tra le parti sia con intese tra le autorità competenti dello Stato e la Conferenza episcopale italiana".

L'Accordo stesso, dunque, stabilisce che le modalità della futura normazione saranno definite tramite "sia con nuovi accordi tra le parti sia con intese" fornendo delle indicazioni, seppur sommarie, alle autorità competenti. Resta fermo, quindi, che la bilateralità pattizia derivanti dall'art. 7 riguardi soltanto $\mathrm{l}^{\prime}$ Accordo e le Intese. L'intesa del 2020, al pari di altri atti similari che assolvono una funzione generica di libera cooperazione, hanno valore giuridico autonomo e sono soggette alla disciplina prevista per l'atto normativo che li immette nell'ordinamento civile ${ }^{13}$.

Alcuni elementi indicati nella raggiunta intesa sul concorso per insegnanti di religione presentano delle difformità rispetto al quadro normativo e, in qualche caso, un netto contrasto con esso; si tratta, per lo più, di elementi ordinariamente normati dall'esclusiva competenza dell'apparato statale. Qui di seguito se ne offre riscontro.

\section{3 - La certificazione dell'idoneità diocesana}

L'art. 4 dell'intesa tocca un punto nodale, direi essenziale, dell'IRC: l'idoneità diocesana. In esso leggiamo:

governo locale, il Mulino, Bologna, 2007, pp. 453-473. Cfr. anche E. VITALI, A.G. CHIZZONITI, Diritto ecclesiastico. Manuale breve: tutto il programma d'esame con domande e risposte commentate, Giuffrè Francis Lefebvre, Milano, 2021, pp. 17 s.; M. D'ARIENZO, L'Intesa e la precarietà, in Il Regno, 2021, 4, p. 81.

${ }^{13}$ Cfr. C. CARDIA, Manuale di diritto ecclesiastico, il Mulino, Bologna, 2001, p. 244; cfr. A. RUGGERI, Intese "concordatarie" e intese "paraconcordatarie" nel sistema delle fonti, in Il diritto ecclesiastico, 1988, 1-2, p. 63. 


\begin{abstract}
"Tra i requisiti di partecipazione alla procedura concorsuale è prevista la certificazione dell'idoneità diocesana di cui all'art. 3, comma 4, della legge 18 luglio 2003, n. 186, rilasciata dal Responsabile dell'Ufficio diocesano competente nei novanta giorni antecedenti alla data di presentazione della domanda di partecipazione".
\end{abstract}

Risulta utile, per meglio comprendere, distinguere il certificato di idoneità, richiesto per partecipare al concorso, dal decreto di idoneità. Il contenuto dei documenti sarà pressoché identico ma il primo è un documento amministrativo, richiesto dallo Stato per una procedura concorsuale statale, il secondo è un documento di esclusiva pertinenza canonica.

Il decreto di idoneità diocesana viene rilasciato dall'Ordinario del luogo, solitamente attraverso i suoi delegati responsabili dell'Ufficio diocesano che si occupa dell'IRC e ha valore esclusivamente all'interno della diocesi che l'ha emesso. Tale decreto attesta che $\mathrm{i}$ docenti siano in possesso del requisito dell'idoneità diocesana ai sensi dei cann. 804-805 del Codice di Diritto Canonico, siano cioè "eccellenti per retta dottrina, per testimonianza cristiana e per abilità pedagogica", e perciò idonei a impartire l'IRC. Non esistono criteri e procedure uniformi per il rilascio del decreto di idoneità in quanto esso resta di esclusiva competenza del singolo vescovo diocesano. In alcune diocesi vige la prassi di indicare nel decreto anche il grado e l'ordine di scuola per i quali il docente è idoneo ${ }^{14}$. Una volta riconosciuta, l'idoneità non ha scadenza, essa è a tempo indeterminato fino al momento in cui non venga esplicitamente revocata dalla stessa autorità che l'ha concessa attraverso un vero e proprio processo canonico, avviato soltanto quando venga accertata "una grave carenza concernente la retta dottrina o l'abilità pedagogica oppure risulti un comportamento pubblico notorio contrastante con la morale cattolica"15. Per completezza va aggiunto che il mancato rilascio del decreto di idoneità non può essere considerato motivo per ritenere l'idoneità riconosciuta come temporanea: nessun docente di

14 Cfr. Delibera della Conferenza episcopale italiana circa il riconoscimento dell'idoneità all'insegnamento della religione cattoliche nelle scuole pubbliche $e$ cattoliche, approvata dalla XXXIV Assemblea Generale, Roma, 6-10 maggio 1991, 2.2 .

15 Cfr. Delibera n. 41 della Conferenza episcopale italiana, approvata dalla XXXII Assemblea Generale, Roma, 14-18 maggio 1990, 2. 
religione potrebbe mettere piede in aula per impartire l'IRC, neppure per un giorno soltanto, se non fosse idoneo, indipendentemente dal fatto di essere in possesso di un decreto di idoneità, poiché il servizio comporta implicitamente la sua idoneità all'IRC. Al pari: la mancata proposta di nomina o il non aver impartito l'IRC per un determinato periodo di tempo non costituisce causa di decadenza dell'idoneità.

Il certificato di idoneità è, invece, un documento amministrativo. $\mathrm{Fu}$ richiesto per la prima volta dalla Pubblica amministrazione in occasione del concorso del 2004 e, come prevedibile, in vista di un bando di concorso, verrà nuovamente richiesto. La ragione della richiesta del certificato di idoneità potrebbe essere la seguente: il concorso è una procedura pubblica di carattere amministrativo con scadenze temporali ben definite. Poiché il decreto di idoneità non ha scadenza e potrebbe esser stato rilasciato molto tempo addietro, lo Stato richiede un documento aggiornato che certifichi che il docente sia idoneo all'IRC, che l'idoneità non sia stata esplicitamente revocata e, molto probabilmente, il grado di scuola in cui il docente è idoneo. L'intesa richiede che tale certificato sia prodotto non oltre i novanta giorni antecedenti alla data di presentazione della domanda di partecipazione.

Se è corretto affermare che il contenuto del certificato, l'idoneità diocesana del docente, sia di pura competenza canonica, è importante anche evidenziare come tale certificato sia finalizzato a produrre effetti in una procedura amministrativa dello Stato. Tale finalità rende inquadrabile il certificato di idoneità come documento amministrativo ai sensi dell'art. 1, primo comma, lett. a), del d.p.r. 445 del 2000. Anche la giurisprudenza segnala la non estraneità del certificato di idoneità all'azione dell'amministrazione. Il Consiglio di Stato (sez. VI) con sentenza n. 6133 del 16 settembre 2000 ha riconosciuto che le norme concordatarie affidano "in via esclusiva al giudizio dei competenti organi ecclesiastici la dichiarazione di idoneità all'insegnamento della religione" e questo comporta "l'impossibilità per il giudice italiano di censurare ex se l'atto dichiarativo in parola". Ma ciò non significa che tale certificato non possa qualificarsi come "atto endoprocedimentale finalizzato all'emissione dell'atto di nomina che resta di competenza 
dell'autorità ecclesiastica italiana"16. In questo caso la finalità consiste in una procedura concorsuale di competenza dello Stato. Gli elementi del certificato di idoneità che non siano di competenza dell'autorità ecclesiale, come ad esempio le tempistiche del rilascio del certificato stesso, sfuggono (o dovrebbero sfuggire) alla competenza ecclesiastica e rientrano nel pieno ambito del diritto amministrativo.

Nella procedura concorsuale del 2004, infatti, tutti gli elementi del certificato di idoneità utili e finalizzati al concorso vennero determinati dal bando, rimettendo all'autorità ecclesiale solo ed esclusivamente il contenuto del certificato: l'idoneità del docente.

Inquadrare il certificato di idoneità come documento amministrativo contribuirebbe, probabilmente, a evitare si possano verificare nuovamente alcuni casi di arbitrarietà accaduti in occasione del concorso del 2004 e che vennero risolti con l'intervento del giudice amministrativo. Non è possibile in questa sede analizzarli tutti nello specifico ma si fa riferimento a quei casi ${ }^{17}$ in cui alcuni Uffici diocesani competenti per l'IRC ritennero discrezionalmente di non rilasciare il certificato di idoneità a docenti di fatto idonei, anche se non più in servizio al momento della presentazione della domanda di partecipazione. Uno degli elementi che accomunò le sentenze, seppur nelle differenze dei gradi di giudizio, riguardò la necessità, in sede istruttoria, di verificare l'effettiva idoneità diocesana dei docenti ricorrenti. I giudici, in conformità a quanto consentito dall'art. 21, settimo comma, della legge n. 1034 del 1971 18 , ritennero opportuno e dovere

${ }^{16}$ Cfr. P. MONETA, Le ultime tendenze giurisprudenziali del Consiglio di Stato in materia ecclesiastica, in Stato, Chiese e pluralismo confessionale, cit., luglio 2010.

${ }_{17} \mathrm{Ci}$ si riferisce, solo per citarne alcuni, alla sentenza del Consiglio di Stato (sez. VI) n. 4860 del 2012 che ribaltò il primo grado (sentenza del T.A.R. del Veneto n. 1482 del 2007), alla sentenza del Consiglio di Stato (sez. VI) n. 6618 del 2007 che confermò il primo grado (sentenza T.A.R. della Campania - Napoli n. 6842 del 2007), alla sentenza del Consiglio di Stato (sez. VI) n. 112 del 2012 che confermò il primo grado (sentenza del T.A.R. della Puglia - Lecce n. 198 del 2009).

18 Il settimo comma recita: "il presidente ha il potere di adottare [provvedimento, N.d.R.] nei confronti di soggetti diversi dall'amministrazione intimata per atti e documenti di cui ritenga necessaria l'esibizione in giudizio". Il provvedimento è stato abrogato dall'art. 4 del d.lgs. n. 104 del 2010 il quale, però, all'art. 63, secondo comma, afferma similmente: "Il giudice, anche 
dell'istruttoria pervenire con certezza all'elemento di natura extrastatuale, in modo da formulare il giudizio con maggiore cognizione di causa.

\section{4 - La riserva dei posti messi a bando}

L'art. 5 dell'intesa riguarda la riserva di una percentuale dei posti da bandire. In esso leggiamo:

"Il 50 per cento dei posti messi a bando nella singola Regione, ai sensi dell'articolo 1-bis, comma 2, del decreto-legge n. 126 del 2019, è riservato al personale docente di religione cattolica, in possesso del riconoscimento di idoneità rilasciato dall'Ordinario diocesano, che abbia svolto almeno tre annualità di servizio, anche non consecutive, nelle scuole del sistema nazionale di istruzione. Ai fini della valutazione dell'annualità di servizio si applica l'articolo 11, comma 14, della legge 3 maggio 1999, n. 124".

Lo scopo di questo articolo è senz'altro quello di garantire che una parte dei posti banditi siano riservati ai docenti precari storici, in possesso, tra gli altri, anche di titolo di servizio. Esso fa riferimento all'art. 1-bis, secondo comma, che si propone la stessa finalità ma la formulazione è differente, non è identica a quella della norma, anche se la differenza è sottile. L'art. 1-bis al secondo comma, infatti, recita:

"Una quota non superiore al 50 per cento dei posti del concorso di cui al comma 1 può essere riservata al personale docente di religione cattolica in possesso del riconoscimento di idoneità $[\ldots]^{\prime \prime}$.

Ordinariamente la determinazione dei posti messi a bando è di competenza statale, coinvolgendo più amministrazioni, quella del Ministero che emette l'atto e, di concerto, il Ministero della pubblica amministrazione e il Ministero dell'economia e finanze che ne autorizza l'avvio definendo il numero dei posti da bandire e la sottile differenza tra l'intesa e la legge potrebbe produrre effetti diversi. Se il legislatore ha previsto che una quota non superiore al

d'ufficio, può ordinare anche a terzi di esibire in giudizio i documenti o quanto altro ritenga necessario, secondo il disposto degli articoli 210 e seguenti del Codice di procedura civile". 
50 per cento dei posti possa essere riservata ai precari con più di tre annualità di servizio, significa l'intenzione di assegnare i posti banditi a tutti i docenti che avranno superato il concorso e si saranno collocati utilmente in graduatoria, poiché se il numero dei precari storici a cui è destinata la riserva dei posti non dovesse essere sufficiente a coprire tale riserva, sarà possibile assegnare i posti ai docenti vincitori non in possesso dei requisiti di servizio previsti per la riserva. Determinare, come invece fa l'intesa, che il 50 per cento dei posti sia riservato ai precari con più di tre annualità di servizio apre alla possibilità che se il numero dei docenti precari che avranno superato il concorso e si saranno collocati utilmente in graduatoria non raggiungesse il 50 per cento dei posti messi a bando, la rimanenza dei posti disponibili potrebbe non essere assegnata agli altri vincitori non in possesso dei tre anni utili per concorrere alla riserva. La graduatoria concorsuale di merito stilata in questo modo potrebbe condurre i docenti esclusi a ricorrere al giudice amministrativo per l'irregolarità della stessa che viola l'art. 15, terzo comma, del d.p.r. n. 487 del 1994 e l'art. 7, primo comma, del d.lgs. n. 59 del 2017, così come modificato dall'art. 1, 792 ${ }^{\circ}$ comma, lett. h), della Legge di Bilancio n. 145 del 2018, norme che prevedono l'assegnazione dei posti messi a bando per i candidati vincitori collocatisi in posizione idonea.

\section{5 - Il programma di esame}

L'art. 7 dell'intesa affronta il punto del programma di esame. Ivi leggiamo:

“Nel rispetto di quanto previsto dall'articolo 3, comma 5, della legge n. 186 del 2003, la preparazione dei candidati è valutata con riferimento ad un programma d'esame comprendente, oltre a quanto previsto nel citato comma 5, anche la conoscenza delle Indicazioni didattiche per l'insegnamento della religione cattolica".

I programmi di esame sono ordinariamente stabiliti dal Ministero ai sensi dell'art. 400, quinto comma, del d.lgs. n. 297 del 1994 sentito il Consiglio nazionale della pubblica istruzione. L'intesa, invece, include nel programma d'esame delle prove 
concorsuali la conoscenza delle Indicazioni didattiche per 1'IRC ${ }^{19}$. A riguardo, in aggiunta, è possibile fare una considerazione più profonda.

L'intesa, all'art. 7, introduce il rispetto di quanto stabilito dall'art. 5, terzo comma, della legge n. 186 del 2003, il quale, oltre a rimandare opportunamente al già citato art. 400, quinto comma, del d.lgs. n. 297 del 1994, specifica che dal programma di esame sono esclusi i contenuti specifici dell'IRC. Una tale disposizione, decisamente insolita considerato che tutti i concorsi per docenti includono la verifica dei contenuti specifici della disciplina per cui si concorre, trova fondamento nell'art. 1, commi primo e secondo, dell'Intesa (sub-concordataria) cui ha dato esecuzione il D.P.R n. 751 del 1985, poi ribadito nel d.p.r. 175 del 2012, che afferma:

"L'insegnamento della religione cattolica è impartito [...] secondo indicazioni didattiche che devono essere conformi alla dottrina della Chiesa [...]. Le indicazioni didattiche per l'insegnamento della religione cattolica sono adottate per ciascun ordine e grado di scuola con decreto del Presidente della Repubblica, su proposta del Ministro dell'istruzione, dell'università e della ricerca previa intesa con la Conferenza episcopale italiana, ferma restando la competenza esclusiva di quest'ultima a definire la conformità con la dottrina della Chiesa".

Lo Stato non ha quindi esclusiva competenza nel definire i contenuti specifici dell'IRC, contenuti che sono il nucleo sostanziale delle indicazioni didattiche. Questa prerogativa esclusiva della Conferenza episcopale italiana fu recepita dalla legge n. 186 del 2003 e anche dalla prima procedura concorsuale svoltasi nel 2004, la quale non incluse la verifica tra le prove d'esame né dei contenuti specifici e né delle indicazioni didattiche.

L'introduzione delle Indicazioni didattiche per l'IRC tra i contenuti delle prove d'esame potrebbe quindi essere considerata una diretta violazione dell'art. 5, terzo comma, della legge n. 186 del 2003 e, indirettamente, dell'art. 1, commi primo e secondo, del d.p.r. n. 175 del 2012. Se la norma non attribuisce allo Stato la competenza esclusiva nel definire i contenuti specifici dell'IRC e le

19 Le intese più recenti sulle Indicazioni didattiche per l'IRC sono state recepite dall'ordinamento italiano con D.P.R. 11 febbraio 2010 (per la scuola dell'infanzia e il primo ciclo di istruzione) e con D.P.R. n. 176 del 2012 (per il secondo ciclo di istruzione e formazione). 
Indicazioni nazionali che li contengono, ne risulterebbe problematica la verifica in sede di prove concorsuali, aprendo la possibilità di impugnare il bando per eccesso di potere ex art. 117 della Costituzione.

\section{6 - La composizione delle commissioni giudicatrici}

L'art. 7 dell'intesa tratta delle composizioni delle commissioni esaminatrici: "Le commissioni di concorso sono costituite ai sensi dell'articolo 3, comma 6, della citata legge [186/2003], tenendo conto di quanto previsto al precedente periodo".

L'articolo richiamato descrive la composizione delle commissioni giudicatrici: presiedute da un professore universitario o da un dirigente scolastico o da un ispettore tecnico e composte da due docenti a tempo indeterminato con almeno cinque anni di anzianità, titolari di insegnamento pertinente con l'accertamento del comma precedente, lo stesso a cui fa riferimento l'art. 7 dell'intesa. Il riferimento al comma precedente riguarda i contenuti delle prove d'esame. In altre parole, l'intesa potrebbe indirettamente indicare che per la composizione delle commissioni d'esame possano essere presi in considerazione $i$ docenti di religione a tempo indeterminato, il che sarebbe una novità assoluta; durante la procedura del 2004 non esistevano, infatti, docenti di religione di ruolo. Ciò ricalca quanto di frequente accade nei concorsi delle altre discipline, ai sensi dell'art. 271, primo comma, del d.lgs. n. 297 del 1994, il quale dispone che le commissioni giudicatrici:

"sono presiedute da un direttore di ruolo o da un docente di ruolo che abbia espletato l'incarico di direzione per almeno cinque anni, ovvero da un docente della materia cui si riferisce il concorso con un'anzianità giuridica nel ruolo di almeno dieci anni e composta da due docenti di ruolo con almeno cinque anni di anzianità nel ruolo, titolari degli insegnamenti cui si riferisce il concorso".

Ciò al fine di poter meglio esaminare i candidati sui contenuti specifici.

A questo riguardo, mettendo insieme le due riflessioni precedenti, quella sulla presenza delle Indicazioni didattiche tra i programmi d'esame e la presenza di docenti di religione di ruolo nelle commissioni giudicatrici, vale la pena fare una riflessione più approfondita. Se in un prossimo concorso per insegnanti di 
religione, venissero nominati membri delle commissioni esaminatrici dei docenti di religione a tempo indeterminato, sarebbe ipotizzabile considerare la valutazione da parte loro dei contenuti specifici e, quindi, anche delle Indicazioni didattiche che li contengono?

Se nella procedura concorsuale del 2004 non esistevano docenti di religione di ruolo che potessero far parte delle commissioni d'esame, ora, a distanza di tanti anni, esistono numerosi docenti di religione che avrebbero tutti i requisiti per farne parte: docenti a tempo indeterminato con almeno cinque anni di anzianità di servizio e titolari del medesimo insegnamento. In altre parole: i docenti di religione di ruolo, non soltanto in virtù dei requisiti previsti dalla normativa statale che consentono loro di prendere parte alle commissioni giudicatrici, ma anche in considerazione del fatto di essere in possesso dell'idoneità diocesana, potrebbero essere abilitati alla verifica dei contenuti specifici dell'IRC? Come docenti idonei di impartire l'IRC e di verificarne l'acquisizione delle competenze nelle aule, tale prerogativa potrebbe estendersi a una commissione esaminatrice in sede di concorso per insegnanti di religione? $\mathrm{Ci}$ troveremmo di fronte a uno scenario inedito che aprirebbe diversi sentieri inesplorati che ci condurrebbero troppo in là.

\section{7 - Conclusioni}

L'intesa procedimentale del 14 dicembre 2020, per quanto ibrida e inedita, sembra rientrare negli atti di mero concerto tra le parti e finalizzata a procedure amministrative che rientrano nell'ambito delle prerogative dell'ordinamento civile, e perciò non collocabile sotto la copertura costituzionale di cui all'art. 7 .

Gli elementi di difformità evidenziati rispetto al quadro normativo riguardanti la certificazione di idoneità, la riserva dei posti banditi, i programmi di esame e la composizione delle commissioni giudicatrici potrebbero destare delle perplessità, come si è cercato di evidenziare, nella prospettiva di un bando di concorso ancora tutto da definire e nel quale gli elementi suddetti riceveranno un evidente peso specifico. 